\title{
Baroque
}

\section{Théorie de l'image et pratique iconologique}

Jean-Marie Wagner

\section{(2) OpenEdition}

\section{Journals}

Édition électronique

URL : http://journals.openedition.org/baroque/520

DOI : $10.4000 /$ baroque. 520

ISSN : 2261-639X

\section{Éditeur :}

Centre de recherches historiques - EHESS, Éditions Cocagne

\section{Édition imprimée}

Date de publication : 1 mai 1980

ISSN : 0067-4222

\section{Référence électronique}

Jean-Marie Wagner, «Théorie de l'image et pratique iconologique », Baroque [En ligne], 09-10 | 1980, mis en ligne le 15 mai 2013, consulté le 30 avril 2019. URL : http://journals.openedition.org/ baroque/520 ; DOI : 10.4000/baroque.520

Ce document a été généré automatiquement le 30 avril 2019

(c) Tous droits réservés 


\title{
Théorie de l'image et pratique iconologique
}

\author{
Jean-Marie Wagner
}

\section{Communication}

1 Je vais revenir sur ce que l'on appelle depuis deux jours l'aspect platonicien du baroque.

Je vous avoue que dans le cas précis je préférerais le qualifier de « sécentiste $»^{1}$, peut-être parce que dans un Centre du Baroque j'ai quelque scrupule à l'appeler "maniériste", peut-être aussi parce que cela n'a pas pour moi une importance majeure : je suis heureux quand j'ai l'impression d'avoir à peu près compris ce que j'ai devant moi et c'est parce que je m'efforce de régler mes problèmes ponctuellement, et de préférence avec les théories explicatives de l'époque, s'il y en a, et nécessairement chaque fois qu'il y en $\mathrm{a}^{2}$. Je m'en vais vous présenter ce qu'est la théorie de l'image, quels sont ses fondements philosophiques, et ils sont connus ${ }^{3}$, et comment tout cela débouche sur une pratique iconologique.

2 Il n'est pas nouveau de voir dans le problème de l'expression le centre de gravité de l'anthropologie philosophique du Cinquecento ${ }^{4}$. Il n'est pas nouveau non plus de voir quels sont les présupposés psychologiques, métaphysiques et éventuellement théologiques qui les sous-tendent ${ }^{5}$, pas plus, d'ailleurs, qu'il n'est original de cerner cette anthropologie en focalisant le débat sur le concept d'image ${ }^{6}$.

3 Car celui-ci est bien sous quelque terme qu'on le nomme, qu'on rappelle «imago», « figura » ou " pictura », qu'on rappelle « species », « effigies » ou « simulacrum », qu'on l'appelle « exemplar» ou «idea », «forma » ou « configuratio », ou encore «notia » ou « intentio », ou « disegno » ou « discorso » ou « concetto » et ainsi de suite, il est bien le «terminus a quo » et « le terminus ad quem » de la connaissance telle que l'entendaient les Renaissants ${ }^{7}$. Aussi le retrouvons-nous à tous les stades du processus cognitif dont l'intérêt ne réside pas dans sa ressemblance superficielle à la démarche éidéique du $D e$ Anima qui fournit, si je puis dire, le fonds de roulement aristotélicien, avec un certain 
nombre de notions - encore que leur usage et partant leur définition soient flottants avec, si vous voulez, aussi et surtout une certaine division du travail de l'« anima » ; bref, c'est moins cet aspect qui importe dans le cas d'espèce que cette pointe platonicienne nuancée par saint Thomas ${ }^{8}$ sans laquelle la Renaissance ne serait décidément pas ce qu'elle est, et qui se perçoit dans l'étude de l'« exemplum » au regard de l'exemple banal, ou de l'imitation servile au regard de l'imitation authentique, ainsi que de l'amour vulgaire au regard de l'amour céleste 9 .

C'est dire, et peut-être ne l'a-t-on pas toujours souligné avec assez de détermination, que l'image est une des meilleures voies d'accès non seulement $\mathrm{au} \mathrm{XVI}^{\mathrm{e}}$ siècle mais encore à l'ensemble du phénomène Renaissance. Carlo Marsuppino dans l'Oratio Quinta caput IV du Commentaire sur le Banquet, nous montre en quelques lignes son rapport à l'immatériel : elle est « exemplar » ou « idea » dans l'intelligence angélique, « ratio » ou « notio », dans l'âme, « imago » ou «forma » dans la matière. Aussi descend-elle du rayon de la « divina potestas» sous la forme d'une "mundi dispositio» et d'un «mundi ordo", véritable «mundi pictura » qui est la marque de la face de Dieu dans le monde, le reflet et la représentation du «mundus » à partir de laquelle il est donné à l'homme de recréer le monde ; représentation lumineusement claire dans le miroir de l'intelligence angélique, plus obscure dans le miroir de l'âme et très obscure dans le miroir de la matière, elle nous apprend que la «materia » est un « obscurissimum speculum » que l'« anima » n'éclaircit qu'en discernant les «formae » qui s'y reflètent, et qu'elle ne saurait le faire qu'en contemplant dans son miroir propre la «splendor divini vultus». C'est donc clair : tout commence et finit avec l'image. L'être sous la forme d'une image est dans le paraître tout comme il est dans le connaître. Dès l'instant où le corps est l'image ombrée de l'âme, le débat culmine nécessairement dans l'intensité, dans l'acuité du regard, de même que dans la nature du regard. La «vis fecunda» du rayon de la «divina potestas» dans ses réverbérations successives et ordonnées donne à l'homme la puissance d'être dans le monde. De miroir en miroir court le rayon qui me permet de percevoir figures et couleurs sous réserve que j'apprenne à voir. Entre l'image intérieure de mon être et son image extérieure, il y a ce vide de la déperdition d'un être dans l'existence et comme une vacuité pleine de bruit et de fureur dans laquelle doit s'inscrire une existence qui se reprend dans l'être en pensant son image extérieure, sur le modèle de son image intérieure qu'elle découvre dans la perception d'un idéal d'être. La théorie de l'« exemplum» nous le démontre à sa façon.

5 L'affaire est plus sérieuse qu'il n'y paraît. «L'humanitas » et la « virtù » s'y profilent en filigrane, et derrière elle l'immortalité. Qu'on se souvienne de l'emploi que Pétrarque fait de l'«exemplum $»^{10}$. Son rôle, à condition que l'« exemplum» soit «verum», est d'« illustrare », c'est-à-dire d'exciter en moi la meilleure part de moi-même, de m'inciter en somme à devenir ce que je suis, de «movere » ma passion non vers l'imitation ou vers le désir d'égaler ou, qui sait encore, de surpasser l'« exemplum ", mais de mobiliser en moi - car ce n'est pas facile - grâce à l'« autoritas » d'une image exemplaire, d'une image que j'ai choisie, d'une image que j'ai bien choisie sous peine de rater mon existence, d'aiguillonner en moi ce vouloir être au bout duquel s'actualise la " virtù ", se réalise un homme et s'affirme une vie dont l'« exemplum » m'avait précisément offert un modèle dans la multitude des modèles possibles tout en m'éveillant - e-moverando- de la médiocrité de mon « habitus »" ${ }^{11}$. C'est en ce sens que l'« exemplum » n'est pas une simple « imago » comme la statue du sculpteur est l'image du corps; elle est bien plus; elle est l'« imago » d'une vertu; elle est «imago virtutum » car sans cela comment saurait-elle 
m'« emovere » au point de me jeter dans la réalisation de ce que je ne connais pas encore comme étant mon modèle propre?

Il n'est donc pas surprenant que près d'un siècle et demi plus tard la thèse d'un Bemho ${ }^{12}$ qui était d'avis que pour parler avec l'éloquence des grands orateurs de l'Antiquité latine, il fallait les lire, les étudier et tenter de les imiter en tous points afin d'avoir au bout du compte quelque chance d'atteindre la maîtrise d'un Cicéron, il n'est pas étonnant que cette thèse soit battue en brèche par son ami Gianfrancesco Pico sur des bases que Pétrarque n'aurait sans doute pas reniées. Selon l'auteur de l'Examen vanitas doctrinae gentium et veritatis christianae disciplinae, libri VI, ne saurait en effet acquérir l'éloquence cicéronienne que celui qui porte déjà en lui l'idée de l'éloquence. Puisqu'il est absurde de croire que l'on puisse chercher et désirer quelque chose qui nous est totalement inconnu et que nulle est la réponse qui repousse la question à l'infini ${ }^{13}$, il faut bien admettre en moi cette «species » du bien dire sans laquelle l'exemple d'un orateur aussi conséquent soit-il, ne saurait déterminer en moi la « dicendi propensio ». Imiter, oui par conséquent, mais non pas imiter tel ou tel rhéteur, mais imiter l'image du « recte loquendi » qui se trouve en moi car c'est elle qui me pousse à lire le Pro Murena ou le Pro Roscio ou tout autre discours ou ouvrage théorique de Cicéron parce que là est ma pente et parce que là elle trouve matière à s'exprimer. Voir Cicéron donc mais pour mieux me voir moi à travers lui ; entre ma perception des écrits cicéroniens et l'image du bien dire que je porte en moi, il y a la complicité de la pluie qui, en arrosant la terre, finira par apporter au germe sa fleur. Mais il y a plus; entre l'image intérieure qui n'a rien de sensible et l'exemple que je perçois, c'est l'image qui me conduit dans ma quête de l'éloquence et m'incite à juger de ce qui dans l'art oratoire d'un Cicéron me convient, preuve de plus, s'il en est besoin, qu'il ne s'agit pas pour moi de devenir un second Cicéron, mais de devenir l'orateur que je suis. Pour ce faire, il est sensible qu'il ne me faudra jamais perdre de vue la « recte loquendi species ", puisqu'elle est la condition nécessaire de ma vertu d'éloquence.

Dans le même ordre d'idée, et sur un terrain où le débat est encore plus connu, se situe la question de la Vénus vulgaire et de la Vénus céleste ${ }^{14}$. Là aussi, bien sûr, il faut connaître pour aimer mais non point de cette connaissance dont nous gratifie un examen rationnel, mais de celle que nous offre le « viso $»^{15}$, pas n'importe quel « viso » cependant : le « viso corporale ", source de l'amour vulgaire, de l' "appetito di belezza sensibile", ne saurait se confondre avec le "viso incorporale » qui seul cause "l'amore céleste », lequel est "desiderio intelletuale di ideale belezza", selon les formules souvent citées de Giovanni Pico. Bref, il y a voir et voir ; voir par les yeux, voir par le sens commun, ce n'est pas « voir vraiment », ce n'est pas vraiment voir, car pour « voir vraiment » il faut voir par le moyen de l'«intelletto». Notons de plus que l'acte de voir par les yeux est dépendant de l'acte de voir par l'« intelletto ", dans la mesure où la puissance de voir par l'intelletto détermine la puissance de voir par les yeux ${ }^{16}$. De la sorte, on comprend mieux que s'exercer à «voir vraiment » revient en fait à apprendre à voir, à travers le «viso corporale », le «viso incorporale» puisque le regard de l'«intelletto» est un «intendere ", on dira aussi un « intueri », qui donne à l'objet de notre perception sa "forme exemplaire », les « ideae » quant à elles, à l'image de "l'idea della belezza idéale », étant des « forme esemplari delle nature delle case e d'esse è pieno agni intelletto e per qu'elle intende $»^{17}$. C'est affirmer on ne peut plus nettement, d'une part, que les «forme » sont en nous et, d'autre part, que les «forme » ne sont pas des images immatérielles d'objets sensibles puisqu'elles donnent précisément de par le regard de l'« intelletto » aux choses sensibles leur « natura », non seulement dans le cas d'une passion telle que l'amour, mais 
encore dans tous les domaines de notre action. C'est pourquoi voir vraiment un objet, c'est apporter à cet objet sa forme, et nos actes ne sont jamais que l'imitation d'une forme que nous portons primitivement en nous. En ce sens, la puissance de voir est aussi puissance de créer, c'est-à-dire d'imiter les images exemplaires que Dieu a déposées en nous, car si d'aventure il n'en était pas ainsi comment expliquerait-on le cas de Praxitèle sculptant son Aphrodite sortant de l'onde en regardant Phryné, son modèle, et de plus célèbre hétaïre s'il en fut !

Qu'il y a voir et voir, M. Ficin nous l'apprend dès le premier degré de l'ascension de «l' animus ad spiritum » dans le $8^{\mathrm{e}}$ livre, chapitre $\mathrm{I}^{\mathrm{er}}$ de sa Theologia Platonica. Si, en effet, Socrate voit Platon, il ne le voit que lorsqu'il touche par les yeux («attingit per ocules») « l'incorporale Platonis simulacrum », et séparée de la matière de Platon (absque Platonis materia), et à cette condition, et elle est capitale, que son œil ne voie pas Platon d'une autre manière que lorsque le corps lui-même de Platon est présent (ut oculos Platonem non videat aliter quam corpore ipso Platonis praesente) ${ }^{18}$.

9 Qu'est-ce à dire sinon qu'il s'agit de toucher, à travers notre perception corporelle de Platon, son simulacre incorporel ; qu'est-ce à dire sinon qu'il s'agit d'effacer sa présence réelle et de le voir comme une absence sensible qui est une présence vraie que seul un regard pénétrant peut atteindre et que l'« intellectus » saura élever après le traitement préalable de « l'imaginatio interna » d'abord et de la « phantasia » ensuite, au rang d'une idée où apparaîtra « la ratio communis » qui lie Platon à Antisthène et à Xénophon, et qui a nom humanité !

10 Il est donc important de voir une présence sur le modèle d'une absence, car la présence n'est qu'une apparence aussi longtemps que l'œil ne s'exerce pas à regarder en perspective en apportant à sa perception la dynamique de la profondeur qui est épaisseur sensible ; non pas opacité qui renvoie, mais matière translucide qui permet au regard de pénétrer le monde des figures avant de remonter l'ordre des miroirs. L'iconologue se doit d'en tenir compte car, dans le cas contraire, que verra-t-il de l'œuvre à interpréter sinon précisément ce qu'il ne doit pas voir pour « voir vraiment »!

11 Cela suppose, il est vrai, que l'œuvre ait cette profondeur - non psychanalytique cela va de $\operatorname{soi}^{19}$ - sans laquelle l'iconologie ne serait au mieux qu'une iconographie, bref qu'elle soit de cette espèce - et à la période qui nous intéresse il y en a - qui ne se livre pas d'emblée au premier venu dans la mesure où son apparence cache une réalité sans que l'on sache très bien, tout au moins "a priori », s'il en est ainsi parce que le sens de l'œuvre vous échappe ou parce que l'œuvre a été créée de telle façon que son sens vous échappe, bien que vous soupçonniez tout de même d'emblée qu'un sens que l'on vous cache est un sens que l'on vous invite à chercher, et que vous pourrez le trouver.

Or, si le sens de l'œuvre vous échappe, c'est, avons-nous souligné, parce que vous ne savez pas la regarder avec "l'œil de l'âme ». Mais, me direz-vous, comment "voir vraiment " l'œuvre d'un autre? Si l'artiste en créant à l'image de tout homme qui agit, comme nous l'avons aussi noté, crée en regardant l'idée de sa création au point qu'elle conduise jusqu'à sa main s'il est sculpteur ou peintre et ainsi de suite, comment pourrions-nous retrouver une idée qui ne nous appartient pas?

Et d'abord, est-il exact d'affirmer qu'elle ne nous appartient pas ? L'idée d'humanité n'estelle pas un bien commun à Platon, à Antisthène et à Xénophon? Si l'idée n'est pas ce concept que notre entendement conçoit puisqu'elle est capable de saisir l'être individuel en soi, c'est-à-dire de l'appréhender tout à la fois dans sa forme la plus riche de 
déterminations singulières et la plus universelle, en un mot de toucher à l'essence propre de cet individu qu'est Platon, d'atteindre son type ou son modèle universel, n'est-ce pas la preuve que «l'intellectus» est justement ce regard qui me permet de contempler les « intelligibilia » comme étant nécessairement " universalia », et donc d'être en mesure de contempler telle ou telle idée de concert avec tel ou tel homme? Il n'y a donc pas d'obstacle spéculatif. Y a-t-il alors un obstacle pratique ? Oui, il y en a même plusieurs : le premier est un obstacle psychologique, le deuxième relève de l'organisation de l'œuvre, les deux étant par ailleurs liés à la finalité de l'œuvre.

Comment arriver en effet à susciter chez un spectateur ce «regard de l'âme » lorsqu'il n'est plus sensible ou lorsqu'il n'est pas encore sensible à la beauté idéale ? Comment faire en sorte que son regard s'accroche à la matière sensible et y pénètre ? Comment lui faire prendre conscience que le monde qu'il a devant les yeux est admirable ${ }^{20}$, qu'il est la représentation sensible d'une "idée», d'un "disegno interno» comme préféra le nommer Zuccaro dans L'Idea de'Pittori, Scultori et Architetti, et d'un « disegno » qui dans ce cas est « divino»? Quand on ne voit pas ou quand on ne voit plus alors que l'organe de la vue est intact, ne peut-on pas apprendre ou réapprendre à voir que le monde et l'homme dans le monde sont le signe de Dieu ? C'est tout le problème. Aussi bien, pensa-t-on que pour le résoudre, il fallait d'abord se donner l'exercice de retrouver le signe de l'homme dans son œuvre conçue - et nécessairement conçue d'ailleurs - à l'image de l'œuvre divine.

De fait, le « disegno interna humano » a bien toutes les caractéristiques de l'« idea » ou de la «forma» telles que nous avons pu les remarquer jusqu'à présent. Il est bien «Ma forma spirituale formata nell'intelletto moi $»^{21}$ qui représente la nature et la forme de tout être dans laquelle l'«intelletto » voit et connaît "chiaramente» un être non seulement dans sa singularité mais encore dans son universalité. Il est même «l'étincelle de la divinité en nous », la marque de Dieu en nous ou, selon le célèbre jeu de mot que l'on rappela hier, le «Segno di Dio in noi » (DI-SEGN-O) ${ }^{22}$. C'est pourquoi, si nous savons en user en créant en tout ou en partie un univers artificiel à l'image de Dieu qui en contemplant dans son propre miroir l'idée immanente à son intellect, créa les êtres et les objets naturels, nous deviendrons ou redeviendrons capables de retrouver la marque divine dans la matière du monde tout comme nous ressaisirons dans le vif notre humanité et affirmerons notre excellence. L'enjeu est donc certain. Il y va de la dignité de l'homme, de sa « virtù » et de sa volonté de jouer le rôle que Dieu lui a confié en lui donnant les moyens de la réussite et la liberté d'en user pour sa perte. Que l'homme fasse la bête, et le monde tombera en quenouille en le projetant dans l'incohérence de la métamorphose. De lui dépend la stabilité du monde : sa fonction est d'ordiner le monde sur le modèle de l'ordination divine qui, si elle est contemplation de l'Idée, est aussi pouvoir de lier les extrêmes. En somme, ce qui est en question, c'est la capacité de l'homme à recréer le monde en contribuant de manière décisive, compte tenu de sa médiane position, à lier l'ordre matériel et l'ordre spirituel ${ }^{23}$. Cette capacité repose sur sa volonté de garder vivant, dans et à travers son corps, le regard de "l'intellectus " afin que soit vraie la formule qui fait de lui le plus beau des monstres ${ }^{24}$ et que soit magnifié à travers lui l'admirable pouvoir de nouer ensemble les contraires sans passer par les moyens, admirable pouvoir dont use Dieu chaque fois qu'il affirme avec éclat sa marque dans le monde ${ }^{25}$. L'importance de l'enjeu est par conséquent telle qu'elle ne saurait tolérer un être humain tout entier livré à ses sens, pas plus d'ailleurs qu'elle ne saurait souffrir la 
médiocrité d'un «habitus» dont la représentation, si elle n'est pas nécessairement bouchée par l'action, est pourtant figée en représentation permanente.

À pareille situation il convient de rétorquer avec force. On répondit en effet en se tournant du côté des passions. Or qui use des passions dans le domaine de l'expression, en dehors de la propre expression de toute passion, fait appel à la rhétorique. C'est très exactement ce qu'on fit. Comme pour «movere » il faut d'abord " emovere », on choisit d'étonner; encore n'y trouva-t-on nul artifice car pour les raisons métaphysiques et théologiques dont nous venons de parler, il est clair que, même mis à part tout subjectivisme, un Zuccaro ne pouvait que mesurer la valeur des différents « disegni » selon l'ordre décroissant de leur l'intérêt psychologico-esthétique et métaphysicothéologique. Aussi est-ce fort logiquement que le « Disegno esterno prodottivo, discorsivo fantastico » remporte la palme, suivi du « Disegno fantastico artificiale » et du « Disegno artificiale » tandis que le « Disegno naturale » ferme la marche.

Est-ce à dire qu'au moment où le spectateur est étonné, qu'au moment où de spectateur il devient acteur selon un processus qui se retrouve même dans la pointe la plus banale puisque c'est par l'idée, et par l'idée seulement que s'effectue le passage entre les deux pôles de la métaphore ou de la comparaison ${ }^{26}$; est-ce à dire qu'au moment où le spectateur devient créateur d'un lien artificiel «quasi imitando Dio ed emulando la Natura $»^{27}$, lien qui, peut-être, est le premier maillon d'un «nuovo Paradiso in Terra » selon l'expression de Zuccaro ${ }^{28}$; est-ce à dire que le spectateur voit ce qui ne peut pas se voir avec « les yeux du corps ", de sorte que c'est à bon droit qu'il nous faut comprendre que rien ne se passe, que rien n'est possible sans la participation d'un « ingegno » qui, s'il n'est pas un « ingegno ingegnoso ", fera en sorte que rien ne bouge en profondeur vu que de toute façon rien ne peut bouger en surface, le mouvement de «l'ingegno ingegnoso » n'étant pas un mouvement des sens mais un mouvement de l'âme ? Est-ce à dire qu'avant que le spectateur ne soit surpris par la rencontre de deux contraires, il ne vivait pas avec ce qu'il voyait, soit parce qu'il ne le voyait pas ainsi, soit parce qu'il ne pouvait pas le voir ainsi, soit encore parce qu'il n'avait aucune chance de le voir ainsi ? Ou bien est-ce à dire aussi que, maintenant que son étonnement témoigne de sa perception de la présence extraordinaire de deux contraires, il voit nécessairement avec « les yeux de l'âme »? Cela n'est pas sûr, bien au contraire, car encore faudrait-il qu'il voie ce qui lie leur présence contradictoire sur le modèle d'un ordre supérieur que seule son «idée ", qui est aussi "l'idée» de tous les «ingegni ingenosi», qu'ils soient «ingegnosi» de nature ou « furiosi » ou « esertati » ${ }^{29}$, qui est aussi l'idée de Dieu, soit parce que Dieu l'a déposée « ab ovo » en eux, soit parce qu'ils imitent Dieu liant les êtres de l'univers, ce qui revient bien à imiter ce que l'on a en soi car sinon, comme nous l'avons vu, on n'imite pas vraiment, encore faudrait-il que notre spectateur comprenne (intendere) grâce à «la forza dell'intelletto ", selon l'expression révélatrice d'un Tesauro ${ }^{30}$, le lien qui unit ce Qui en apparence ne s'unit pas. Si l'étonnement est source de la «maraviglia» et si la «maraviglia » est cet instant où le sol de la réalité semble se dérober sous mes pieds tant l'apparition est stupéfiante au point que je me retrouve tout d'un coup comme désorienté, comme étranger dans un monde que je ne connais plus alors que je croyais pourtant le connaitre, que vienne à me manquer alors cette «maravigliosa forza dell'intelletto » et me voici plus que jamais soumis à la volonté de mes sens extérieurs, non plus livré au morne train-train de mon "habitus» d'avant l'instant de mon étonnement, mais bien au contraire agité au point de passer sans cesse en revue sans rime ni raison, et souvent non sans plaisir, les figures d'un « disegno estemo prodottivo, 
discorsivo fantastico», d'un «disegno fantastico artificiale» ou d'un «disegno artificiale " pour au bout du compte conclure au côté spectaculaire de ce que je vois et à coup sûr à l'incohérence de ce que je vois et parfois à la folie de l'auteur qui a créé ce que je vois. Or, s'il en est un qui dans cette affaire est fou en effet, ce n'est pas lui mais moi, non pas fou de folie naturelle mais fou de folie par opinion, cette folie fausse dont parle.

P. de L'Ancre qui est le signe clinique d'une authentique maladie de l'âme ${ }^{31}$.

Et c'est ici que nous rencontrons notre deuxième obstacle, celui qui relève de l'organisation de l'œuvre. Il arrive en effet, et comment cela n'arriverait-il pas dans ce contexte, que l'auteur s'ingénie à me compliquer la tâche non par goût du vice, mais parce que ce faisant il donne à mon « ingegno » l'occasion d'être « ingegnoso » comme il se donne l'occasion de faire preuve d'ingéniosité et de soulever ainsi l'admiration et les applaudissements de tous ceux qui viennent regarder son œuvre. De même se donne-t-il encore à lui, l'occasion d'imiter Dieu, et à moi, l'occasion d'imiter l'Homme, et à nous deux, l'occasion d'être "humaniores" dans une "concordia figurarum » d'êtres et de choses. Suivant l'esprit d'un jeu de la découverte dont il est seul à connaître la vérité, il hermétise donc son propos car il y va de sa gloire, de son immortalité aussi, comme il y va de la mienne dans la mise à l'épreuve de ma capacité de déchiffrer notre commune humanité dans une œuvre humaine imaginée sur le modèle de l'œuvre divine. Dans tous les cas il souhaite et toujours tentera, par les moyens artificieux les plus divers, d'exciter, de provoquer en moi le désir de trouver le sens, fût-ce au prix de l'énigme, et souvent non sans accumuler à dessein tous les obstacles à ma perte. Et, pour peu que le jeu prenne la dimension d'un tableau de la vie, la qualité ludique de la question, surtout si elle est figurée ou mêlée de figures et de mots, ainsi que la qualité de l'interrogation et de la réponse viendront construire le rite d'une initiation en mettant en jeu l'existence d'un mythe, c'est-à-dire en mettant en question ma propre existence au-delà de celle du spectateur que j'étais ou de celle de l'iconologue que je suis encore tout en ne l'étant plus tout à fait, puisque je suis forcé d'être moi-même. Car c'est à moi qu'il appartient de comprendre le sens de ce que je vois; c'est à moi spectateur de devenir en somme un iconologue avec cette nuance toutefois que le jeu que je joue, dépasse de loin le processus d'une classique interprétation iconologique puisqu'il y va de ma "virtù », puisqu'il y va jusqu'à ma capacité de ressaisir mon essence dans mon existence, puisqu'il me faut ni plus ni moins re-créer un «Paradiso in Terra » qui ait la clarté du Jardin de Jupiter, puisqu'il me faut voir dans la réalité que je perçois tel un puzzle dans le jeu complémentaire d'une rhétorique de la dissuasion et d'une rhétorique de l'argumentation figurée qui est un "discorso esterno " conçu sur le modèle d'un "discorso interno ", puisqu'il me faut «viser» dans cette réalité morcelée la dialectique d'un sens. Or saisir cette complémentarité revient à maîtriser le mouvement centrifuge de la dispersion de mon être dans l'apparence d'un univers étonnant au profit d'une dynamique de l'unité qui est dynamique unitaire de mon «ingegno ingenoso ». Celui-ci est d'abord, mais du point de vue du créateur, fulgurance métaphorique, fulgurance métaphorique artificielle sur le modèle de la fulgurance métaphorique naturelle de Dieu avant d'être, mais, du point de vue du spectateur cette fois, d'un spectateur qui en devenant acteur s'est retrouvé à son tour créateur en recréant sur le mode d'une co-naissance, sur le mode d'une participation au «disegno interno » du créateur initial, l'œuvre que celui-ci a en partie créée dans ce but, avant d'être transparence pensée d'un discours de l'être dans le monde. Saisir cette complémentarité entre la rhétorique de la dissuasion et la rhétorique de l'argumentation figurée revient donc aussi à saisir le sens de l'œuvre, dans la mesure 
où la rhétorique de l'argumentation figurée n'est que la représentation figurée de la pensée de "l'ingegno ingenoso " mais toujours sur le modèle de "l'ingegno divino ». D'ailleurs Dieu ne m'a-t-il pas donné un «intelletto » qui est un miroir dans lequel viennent se refléter les images du monde extérieur? Et n'est-ce pas dans ce reflet immatériel des objets du monde que je les perçois, avec sans doute l'inévitable concours du mot $^{32}$, de sorte que ma compréhension du monde, ma perception du monde même, est nécessairement un « nodus » d'images et de mots !

Quoi qu'il en soit, saisir le sens, on ne le peut qu'en disposant son âme de manière à la mettre en mesure d'«attingere", dans le «disegno» ou "discorso estemo», le « disegno » ou « discorso interno ». À l'« animae dispositio » répond la disposition du monde qu'elle a recréé, de même qu'à la disposition du monde répond comme dans un miroir la disposition de l'« anima » créateur.

Entre l'«anima» créateur et l'«anima» re-créateur il y a au terme du processus recréateur cette même complicité du regard qui est mouvement regardant la même « idea » à travers une matière figurée. C'est pourquoi il y aura toujours, entre l'« anima ", créateur et le regard incapable de percevoir la présence absente d'une réalité dans la réalité présente d'une absence, l'opacité de la matière qui ne se reflète pas mais qui se perçoit dans un miroir, et dans un miroir de figures. Certes, dans le jeu qui est le nôtre où "l'idée » se donne dans une matière transfigurée comme la trame d'un discours de l'être sur l'être qui instaure un cosmos dans un face à face de la matière et de l'esprit, l'iconologue, au strict sens de ce terme, a sa part. Elle est celle que lui laisse l'auteur dans le processus de la création suivant qu'il utilise en tout ou en partie une symbolique qui est pour l'essentiel donnée et partagée par ses contemporains et consignée dans des ouvrages à l'usage de tous. Il coule de source cependant que dans les cas extrêmes, là où l'enjeu est celui de l'homme, et de l'homme dans le monde, et de l'homme en Dieu, il n'y a jamais totale utilisation d'une symbolique dûment répertoriée. La raison en est simple : elle ne permettrait pas le jeu de l'imitation; elle ne permettrait pas non plus que fonctionne le mythe de la création du monde tel qu'il a pu être perçu, tel qu'il a pu être élaboré autour du thème de l'« homo médius » dans la période de la Renaissance. Faut-il en conclure que dans cc cas en présence d'œuvres aussi précieuses, aussi déterminantes pour une époque, tellement ils la représentent dans ce qu'elle a de plus intimement pensé, il ne faille décidément pas savoir voir au sens physique du terme ? Ce serait évidemment bien léger que de le croire; d'ailleurs notre bon sens le plus élémentaire s'en trouverait heurté. Point n'est besoin d'être philosophe pour savoir que nos sens sont le premier mode de notre connaissance et que pour percevoir il faut d'abord avoir de bons yeux. Mais entendons-nous, il faut tout de même voir avec cette qualité de l'attention qui se pense en mouvement. Qu'on se rappelle l'anamorphose, cette « argutezza » de l'art pictural. Après la «maraviglia » et l'étonnement qui est interrogation des sens sur le sens et déjà impulsion à penser, il y a la recherche du point de vue qui est aussi un point de compréhension. Mais entre l'instant de l'interrogation qui n'est jamais qu'un certain point de vue, et l'instant de la compréhension qui est le point de vue privilégié, il faut savoir regarder avec « les yeux du corps ».

Pour illustrer ma pensée, je ne peux mieux faire que d'évoquer l'exemple suivant ${ }^{33}$ : 

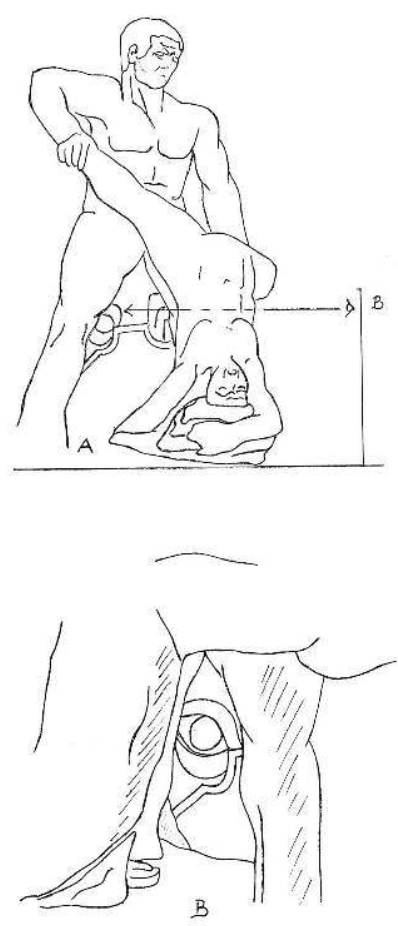

Il est intéressant à plus d'un titre, ne serait-ce que parce qu'il apporte à notre propos l'ironie qui lui manquait ou plutôt parce qu'il est en soi plein d'ironie. Comment en effet l'iconologue ne s'esclafferait-il pas sur lui-même lorsque, parcourant le Bois Sacré de Bomarzo tout au désir de "voir vraiment » après avoir souvent regardé le géant non pas d'en haut - bien qu'il l'ait fait -, ou du haut de l'escalier - bien qu'il l'ait fait aussi - là, et seulement là où l'escalier fait l'angle et nous porte tout droit vers cette figure énorme composée d'un géant écartelant un androgyne, comment n'aurait-il pas pu être stupéfait après l'avoir contemplée maintes et maintes fois avec tout le soin nécessaire, c'est-à-dire avec cette qualité d'une attention dynamisée par le mouvement semi-circulaire de gauche à droite et de droite à gauche que la murette basse construite en arc de cercle qui l'entourait jadis exigeait du spectateur ; comment n'aurait-il pas pu être suffoqué de voir subitement, lui qui s'était longuement interrogé sur le sens de l'étrange signe taillé dans le roc que l'on peut apercevoir en A (fig.1) par l'ouverture à gauche de l'écartelé, comment n'aurait-il pas été interloqué que, dès l'instant où le hasard l'ayant posté en $B$ (fig. 2), et uniquement en $\mathrm{B}$, face à l'ouverture à droite de l'écartelé, une grande partie de la gauche du signe se donnât enfin pour ce qu'elle est, c'est-à-dire pour un œil, énorme lui aussi, qui soudain le contemple à son tour comme lové entre le haut du corps de l'androgyne et la jambe de gauche du géant, bien encadré par ces deux parties de la figure, œil penché sur la gauche et à hauteur du regard! Situation ironique en effet qui illustrerait à merveille la fable du regard regardé. Situation significative aussi qui devrait donner à penser que le Parco di Mastri mériterait un bien meilleur sort.

Pas de doute par conséquent, il faut que l'iconologue ait de bons yeux : car « le regard du corps " prépare « le regard de l'âme », le provoque à " perspicere » comme « le regard du corps » avait lui-même été provoqué par un stupéfiant objet de perception qui a rompu le cours ordinaire et sclérosé de la vue ${ }^{34}$. Or c'est dans cette rupture qui s'ouvre sur le vide, que s'engouffre l'errance livrée aux sens externes. Celle-ci est le plus souvent définitive et toujours joyeuse et distrayante mais non sans danger ${ }^{35}$, ponctuée de surprises qui 
poussent au mouvement du corps, surprises parfois énormes, de ces surprises qui ont littéralement le don de vous frapper de stupeur à l'image des figures bomarziennes. L'errance momentanée est plus rare: trouver le sens d'un non-sens suppose l'intervention d'un «ingegno» qui brise le mouvement du corps au profit d'une dynamique de l'âme. Bref, l'errance ne trouve sa fin que dès l'instant où "l'ingegno " trouve sa verve d'être "ingegnoso ». Celle-ci tient à un pouvoir, celui d'«intendere » "l'idée ligatrice» qui, en liant en réalité ce qui était délié en apparence, lève l'interrogation qui prit racine dans l'absurdité de la vision primitive. De la sorte pourra-ton parler, dès lors que le dessein est assez vaste pour donner lieu à un authentique discours de figures étonnantes, d'une véritable circulation de «l'idea » centrale, c'est-àdire du « disegno interno » animant grâce à l'ingéniosité de mon « ingegno » le « disegno esterno » et actualisant par là même le "discorso interno » dans un « discorso esterno » tel l'« animae oculus » retrouvant dans son miroir propre le « mundi ordo » de la « divina potestas » dans l'« obscurissimum speculum » de la « materia ».

Et nous revoici devant l'enjeu et à même d'en mesurer l'amplitude. Car entre «l'argutezza » la plus banalement humaine et «l'argutezza » divine, il y a les chocs de tous les contraires d'une chaîne brisée ; ils nous parlent du lien de l'âme et du corps, de l'âme et du monde et de l'âme et de Dieu dans le monde, etc., sur le modèle artificiel des rapports d'un «disegno interno humano » avec son «disegno esterno artificiale » ou mieux encore "prodottivo, discorsivo, fantastico », de même qu'ils nous parlent du vide que l'incompréhension crée jusqu'à ignorer ou pire à nier - et quelquefois non sans obstination - l'existence même du "disegno interno » au point d'installer durablement dans le « disegno esterno » la seule compréhension des sens extérieurs. Or croire dans la période maniériste à une matière qui n'aurait pas en son fond la "divina figura », serait s'exposer par ignorance aux mécomptes d'une initiation mort-née. Certes, l'importance de la théorie de l'«idea " peut étonner et peut-être pensez-vous que je lui en accorde trop.

De fait, si l'on entend par importance une grande influence tout à la fois dans l'espace et dans le temps, alors, sans aucun doute, je lui accorde trop d'importance. Mais si on entend par importance la mise en relief de ses fondements philosophiques - lesquels entrent nécessairement en jeu en cas de "cultus apex»-, et qui en font tout de même autre chose qu'un simple exercice raffiné pour bel esprit, alors son importance me parait déterminante, même si, et je vous le concède volontiers, semblable jeu n'est pas et ne sera jamais à la portée du plus grand nombre, et pour cause.

Certes, un de ses buts évidents, surtout si l'on va en quête de ses sources (Aristote, Cicéron, Quintilien, etc.), est d'ordre rhétorique. Il s'agit d'arriver à attirer l'attention de l'auditeur en évitant de parler plat, l'ennui, « la noia », et la torpeur d'un auditoire étant les pires ennemis de l'orateur. Il n'empêche que l'on passerait à côté de l'essentiel si l'on ne retenait du célèbre éloge tésaurien de l'« argutezza » que les propositions - et elles sont fort nombreuses - qui vont dans ce sens ${ }^{36}$; et si l'on ne voyait pas combien déterminante est cette capacité que se donne l'homme parce que Dieu la lui a donnée de lier les extrêmes par les moyens grâce à l'« idée » d'un « ingegno ingegnoso »; car elle est proprement celle de Dieu. Elle permet à travers la participation active d'un spectateur à l'œuvre artificielle mais réelle et dans le meilleur des cas fantastique, productive et discursive d'un créateur, de retrouver l'idée que la théologie chrétienne a développée ; celle-ci consiste à donner à la créature le devoir, dès l'instant où elle porte en elle, à 
l'image de l'homme, la marque de Dieu, de participer à l'ordre de la création sous peine d'un effondrement du cosmos par effondrement de son centre.

On devine alors que par-delà la recherche du "godimento », de l'admiration, de la gloire par les lettres et les arts et ainsi de suite, plaisir fugace et délectable d'aristocrates lettrés, - et il y en eut bien évidemment, il y en eut même beaucoup -, il y a plus fondamental. Sous des dehors parfois précieux, le plus souvent érudits et à coup sûr ésotériques, et en cas d'« exemplum» hermétiques, le message est de ceux qui nous enseignent que pour être, l'homme doit porter le monde sur ses épaules mais dans la main de Dieu.

\section{Discussion}

27 - HALLYN : L'iconologie met donc en abîme toute l'époque ; la science nouvelle de Galilée n'a-t-elle pas supprimé cette conception de l'homme déchiffreur d'images en cherchant à comprendre les lois abstraites du monde?

- WAGNER : La crise, l'effondrement d'un monde ont longtemps servi pour expliquer le baroque ; en particulier les tenants du maniérisme fondent ce genre sur le sac de Rome, l'effondrement du Christianisme et la révolution copernicienne ; les partisans du baroque évoquent la perte du centre du monde. Un nouveau monde mécaniste apparaît au XVII ${ }^{\mathrm{e}}$ siècle chez les auteurs les plus lus. En revanche, les «minores », la littérature banale à grand tirage révèlent l'importance vitale de la démonologie et de la magie.

Tous les hommes n'entrent pas dans l'ère nouvelle dès le XVII e siècle; certains relèvent encore du XVI ${ }^{e}$ siècle; d'autres passent sur l'héliocentrisme de Copernic. Tout le monde n'a pas compris la révolution mécaniste. Pour les gens du siècle, la crise est morale et religieuse; pour nous, elle est scientifique. D’ailleurs, le christianisme va digérer ces nouveautés, y plaquer le diable et faire de la crise scientifique une crise religieuse, une attente de la fin du monde,

30 - Marie-France SALQUES : Koyré insiste, par exemple, sur une poésie de John Donne qui fait allusion à la révolution mécaniste et montre la terre qui perd son centre. Cependant, peu de textes vont dans ce sens.

31 En revanche, le dernier ouvrage d'Elizabeth Labrousse étudie l'éclipse solaire de 1656 et révèle la crainte de la fin du monde,

32 De même certains jardins de Florence, aujourd'hui disparus, révélaient les changements dans les esprits.

33 Les historiens de la philosophie ont donc été fascinés par le travail des historiens de la science, mais ils ont été trompés, car les hommes du temps n'ont pas eu conscience du changement.

34 - Geneviève DUVAL-WIRTH : L'iconologie et les devises s'accommodent pourtant de la révolution mécaniste et montrent, dès le début $\mathrm{du} \mathrm{XVII}^{\mathrm{e}}$ siècle, les instruments et les outils des constructeurs. Dans son traité, Filippo Piccinelli, jésuite, émule italien du P. Ménestrier, mais plus riche d'idées que ce dernier, avoue n'être pas encore parvenu à la totale perfection dans cet art. Les devises, document collectif qui reflète la mentalité de l'époque, centralisent l'univers autour d'un axe; tout tourne autour des pôles et des centres. Cyrano, cas particulier et complexe, parle de deux pôles et de deux centres, avec zone d'ombre, zone de lumière et passage de palier en palier; il arrive au centre de la 
partie lumineuse, passe à travers l'obscurité grâce à un médium qui se présente surtout comme une force imaginative... Aspect paradoxal de ce matérialiste.

- WAGNER: Il s'agit, dans son cas, de la force de l'intellect plus que de la force de l'imagination. Ainsi, Les Ambassadeurs de Holbein, au-delà de l'anamorphose, nous invitent à préciser le sens des instruments posés sur la table. D'autre part, malgré l'existence des dictionnaires, la symbolique est difficile à interpréter et l'iconologie pousse à rechercher la logique interne des images.

36 - HALLYN : Où se trouve le point de rupture entre les deux imaginations que nous distinguons: celle qui existe à partir du réel et celle qui existe à partir de la vie intérieure? Tesauro, pour sa part, fait une création et non une re-création: "Dieu produit ce qui est de ce qui n'est pas ; ainsi l'esprit de ce qui n'est pas, fait ce qui est?» Voyons-nous émerger un nouveau type de création?

37 - WAGNER : Pour l'artiste, il s'agit de l'imagination créatrice et, comme le dit Descartes dans la Première Méditation, les images créent.

38 - HALL YN : La distinction entre création et re-création est peut-être une illusion de la part de Tesauro. En tout cas, elle dépasse les théories sur l'imagination créatrice qui participent de la création de Dieu. L'artifex produit-il tous les objets d'art à partir de rien ou produit-il seulement des monstres?

39 - WAGNER : Cette nouvelle théorie s'oppose aux rationalistes et Malebranche va, par exemple, tonner contre elle. Il s'agit cependant d'un dernier feu d'artifice. En fait, tout revient à l'intellectus, notion artistotélicienne pour désigner cette faculté de l'âme qui évolue chez tous les auteurs : Elle s'applique aussi à la notion de miroir : pour Tesauro, le miroir est comédie. Cependant, Gian Francesco Pico perçoit des images immatérielles, et une chose qui n'est pas imagination, mais imitation.

40 - HALLYN : Alors se pose un autre problème de terminologie: qu'est-ce que le logos? Tesauro emploie un terme nouveau, difficile à traduire : produce. S'agit-il de produire?

41 - Geneviève DUVAL-WIRTH : Pour sa part, Francisco Patrici s'oppose à l'aristotélisme en poétique et favorise la « produzione » qui ne doit pas se manifester de manière déréglée ; il insiste sur les possibilités de la création: engendrer des monstres ou des choses ordonnées.

42 - WAGNER : En effet, quel est le rôle de Dieu quand on met des monstres au monde? Le monstre, présage de la fin du monde, effraie. Les auteurs posent à son sujet des questions physiologiques ; ils montrent le miracle de Dieu ou incitent à craindre la punition divine.

43 - CASTAN : Quel rapport avec l'artiste intérieur de Giordano Bruno?

44 -WAGNER: Giordano Bruno, qui parle du processus de personnalisation de l'idéal platonicien, est encore un Renaissant. D'un point de vue moderne, l'imagination est source d'erreurs. Ainsi, Descartes et Malebranche redoutent la puissance imaginatrice. La conception de l'imagination procède d'un fond théologique et psychologique. Il s'agit d'une faculté investie par le diable. Ainsi Descartes place un malin génie dans le doute méthodique.

45 Cependant, l'imagination est aussi lieu neutre; tout est dans l'intention : le diable, ange déchu, présent par la permission de Dieu pour permettre à notre libre-arbitre de s'exercer, souhaite tromper; il actionne donc l'imagination dans le sens du mal.

46 - HALLYN : Cyrano laisse une place à l'imagination-production dans la création divine. Il joue sur les mots en parlant de feu dans l'inspiration au cours du Voyage dans la lune. 
47

- WAGNER : L'imagination apparaît bien comme un lieu neutre, définissable à la manière de l'image incorporelle de Platon.

Les notions d'absence et de présence justifient l'iconologie ; la perception sensible est source d'erreurs ; les yeux du corps ne permettent pas de comprendre Bomarzo ; ceux de l'âme, si.

- MARTINON : Orsini, l'homme qui a commandé Bomarzo, possède Apollonius de Thyane, « le grand damné » dans sa bibliothèque, mais cet amateur de pastourelles n'a qu'une culture moyenne. Ligorio, qui est peut-être l'auteur du jardin, était un faussaire, pilleur de tombes étrusques, collectionneur de pierres gravées. La signification, peut-être magique, des pierres gravées, reste à découvrir.

- WAGNER : Le songe de Polyphile aurait influencé l'histoire des Jardins de la Renaissance, mais Bomarzo demeure une œuvre hermétique dont aucune œuvre littéraire ne nous donne l'interprétation. Au départ, une recherche sur le terrain à la manière de Mandiargues, se heurte à de grandes difficultés, car ces rochers de tuf bleuté sur fond de prés verts sont rongés par le lierre et très friables.

51 Ensuite, comme devant le Songe de Descartes, vient une perception neuve des jardins, sans préjuger des autres interprétations déjà données.

- Marie-France SALQUES: Le desengaño d'Apollon est au centre du baroque, dans la métamorphose de Daphné. Tapié a montré, déjà, que l'air est un élément de fond dans la sculpture baroque. De même, Le feu des signes, écrit à propos de l'art byzantin, est à utiliser pour analyser le baroque: l'ouverture des verrières, l'arrivée de la lumière triomphent de la pesanteur ; cette dernière sera définitivement vaincue par le rococo.

Les miroirs deviennent élément folklorique ; on les pose sur les vêtements ; ils indiquent le pouvoir d'un être sur une autre personne. Le reflet donne le pouvoir. Comme à Marly, Versailles et Tivoli, le pouvoir donne le reflet et rassemble le peuple dans des fêtes. Cependant, la fête populaire est différente de la fête royale. Le peuple demeure une case vide ; la chaire permet de triompher du vide permanent de la parole ; la messe devient opéra ; le sacré disparaît dans la fête baroque, comme Stendhal à la Scala regardera sans être vu, le théâtre permet de regarder sans être vu.

4 La ville elle-même devient, comme la demeure, une perspective organisée : Richelieu, par exemple, s'oppose à Rome, ville construite sans plan.

- KRYNEN : Des perspectives nouvelles apparaissent avec les recherches de Wagner qui unissent iconologie et théologie. La philosophie éclipse la recherche thématique entreprise à la suite de Jean Rousset, qui s'était laissé prendre au piège des catégories visibles à la surface de l'œuvre.

6 Le baroque n'est pas davantage la catégorie unique exposée par Eugenio d'Ors. Une dualité apparaît, pôles, formes, âme et corps, essence et existence. Les tensions et les relations entre ces catégories changent suivant la situation historique, mais il demeure toujours une situation de rupture verticale, ontologique, entre Dieu et l'homme.

7 Le baroque est volonté de parvenir à une synthèse superficielle ; dans un état d'équilibre instable, l'homme baroque se trouve à la jonction entre l'esprit et le corps.

Une genèse résulte de l'affrontement dramatique de ces contraires, la culture baroque est issue de la rupture.

Production intérieure en dissolution; ainsi, le classicisme sort du baroque, mais pour recommencer un cycle influencé par le baroque. 

d'une situation historique ; rupture spirituelle ; expérience du conflit entre l'homme et Dieu ; volonté de conciliation et stoïcisme inhérent au baroque.

61 Le christianisme, subissant certains clivages, complique l'interprétation. Il convient de distinguer entre théologie catholique et théologie protestante. L'homme en voie de divinisation réalise une promotion du logos dans l'église et dans la culture. Luther, Fray Luis de Leon font surgir une contestation radicale. Discussion générale : Hallyn, Krynen, Jechova, Wagner, Gyenis.

La distinction claire entre baroque éternel et baroque historique, entre baroque et classique est impossible. Il existe des contradictions essentielles entre les différents baroques ; dans le temps, les limites entre baroque populaire et baroque savant ne sont pas identiques. Une recherche interdisciplinaire en situation historique et géographique est indispensable.

\section{NOTES}

Il est en effet intéressant de voir que si l'on n'accepte pas l'existence de cette catégorie on se retrouve avec des hommes comme Pe est seulement de 1655. C'est évidemment embarrassant. Alors on les ignore; ou bien, comme on ne peut tout de même pas 1es ranger d

Toute théorie explicative d'une époque telle que nous entendons cette expression repose à l'évidence sur une conception du monde, c'e , Paris, Gallimard, 1967. p. 22), qu'il soit dans l'obligation de s'assurer de la création et de la logique symbolique, c'est-à-dire de

ce qui revient en fait à reconnaître que le concept, nécessairement conçu par toute catégorie viable, se volatilise dans la pratique histo

4. Cf., par exemple.R. KLEIN :La Théorie de l'expression figurée dans les traités italiens sur les « imprese »,

1555-1612, inLa Forme et l'intelligence,Paris, Gallimard,1970, pp. 129-130.

Il suffit pour les rencontrer de prendre n'importe quel philosophe de la Haute et de la Moyenne Renaissance. Est-il besoin de rappeler q 6. Cf., par exemple, W. RAITH :Die Macht des Bildes,W. Fink Verlag München, 1967.

chaque concept sa spé

princeps), 1, 3. Il n'en va évidemment pas de même pour « idea », « forma » et « exemplar », dont la marque thomiste est extrêmement 
8. Entendons-nous. Il n'est pas question de tempérer la veine néo-platonicienne, bien au contraire. Il convient en fait de dis ne voir qu'un aspect du débat, celui de la création sur le modèle de la conception de l'« artifex ».Dans cette optique, il ser et d'une manière délibérée grâce aux procédés artificieux que nous connaissons -d'égarer son spectateur en vue de susciter une recré -r61toçde l'époque, il lui arrive cependant d'être utilisé dans une perspective franchement platonicienne, par exemple par Giovanni

M.DC.LXX. Per Bartolomeo Zapata, pp. 357 et sq., les mots extrêmement vifs (par exemple « socioccamente ») et les jugements tout aus Idea- Ein BeitragzurBegriffsgeschichte der Älteren Kunsttheorie (B. G. Teubner, Leibzig-Berlin, 1924) -ne sut pas toujours l'« idea » chaque fois qu'il y a bonheur du regard, tandis qu'il ne dépasse qui ne so

9. Cette énumération des différents dom , est celui de l'explication finicienne de la naissance de l'amour et de la haine d'un homme pour un de ses semblables. Plus que jamais, e

10. Cf. la fameuse lettreàGiovanni Colonna ; elle est brièvement analysée par W. RAITH :op. cit., pp. 26 et sq. Colonna avait reprochéàPétrarque d'abuser de l'« exemplum » au point d'altérer la puissance analytique de ses écrits.

11. À comprendre dans le sens aristotélicien.

12. Cf. W. RAITH :op. cit.,pp. 67 et sq.

De deux choses l'une, ou j'ai en moi l'idée d'éloquence (thèse que Pico fera triompher) ou je l'ai reçue du dehors (thèse de Bembo). Si ell

Très nombreux sont les textes sur ce thème. Du fait de son utilisation symbolique et iconologique, nous le trouvons pour ainsi dire dans

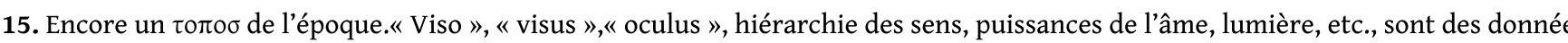
Commento sopra una canzone di'amore(Venezia, 1524 ; trad. française de Gabriel Chappuys,àl , n'est, de ce point de vue, nullement original. Rappelons que pour Platon déjà, l'Idée ou la Forme n'est pas le fruit de notre entendemen

République(X, $596 \mathrm{~d})$ fait un lit

Du point de vue strict de la démarche éidéique, ce n'est plus absolument vrai du point de Vue de la hiérarchie des sens, l'ouïe et la vue

17. GiovannipIco :op. cit.,libro II, capitolo X.

Dans le cas contraire, il ne percevrait pas mais imaginerait. Pour saisir pleinement le sens de ce propos, il convient d'avoir en mémoire «mens »(cf.FICIN :Theol. Plat.,I, 6. Giov. Pico :Heptaplus,V,1).Ficin nous le dit explicitement dans l'Oratio Quinta

l'âme que lorsque son image est captée par la vue ou (vel) conçue (conciptur) par 1'« animus corpus esse non potest ». N'oublions pas, en effet, que pour Ficin 1'« oculus » est « l'animae instrumentum ». Rappelons-nous enfin le $r$ 19. Notre souligner qu'il y a des cas où il convient de savoir s'en passer sous peine de mélanger la chèvre et le chou. D'où l'importance de la déter

la culture d'une époque serait une erreur de méthode qui trouverait nécessairement sa sanction dans son in

20. -Souvenons-nous des remarques de saint Augustin dans leDe Trinitate,lib. III, Cap. II, 7 : « L'ordre naturel est lui aussi, la continuité de l'habitude émousse l'admiration(« perseverentia tamen consuetudinis ami , 11 : « Mais si

la volonté divine) arrivent, pour ainsi dire, avec le fleuve sans fin des choses qui passent, s'écoulent et, par des chemins connus, passen

àl'obscurité, on le trouve tout naturel... Au contraire,si, pour piquer l'attention humaine, ils sont prodigués suivar ( « cum vero admonendjs hominibus inusitata muta 
Notons par ailleurs que dans la perspective néo-platonicienne, le désir de connaissance qui est désir de beauté et désir d'aimer, est toujours admiration de la splendeur divine dans les corps. Admirer la nature, c'est admirer « Porus » en elle, etc.

21. F.zUCCARO :Op. cit.,I, 3.

22. Cf. dans ce numéro l'intéressante communication de F. HALLYN.

23. D'où l'importance- non exclusive cependant-del'EthiqueàNicomaque dans le domaine de la morale et de l'éthique.

24. Expression connue. Elle vient de loin (dans la tradition de Mercure Trismégiste, l'homme homo médius ». L'homme est un monstre parce qu'il est composé de deux natures opposées, l'âme et le corps. L'homme est un beau mo « nodus », sans lequel la chaîne des êtres serait déliée, mais encore une « copula mundi » qui

l'a-t-on parfois qualifié de " petit Dieu », non dans un sens prométhéen, mais dans le sens d'une créature qui, portant en elle la m Corpus Hermeticum(cf.t. I,X, 24-25, de l'éd. Festugière). L'appréciation la plus juste nous est d (notons que le monde et l'hor

Si l'homme assume cet office en tout ce qu'il comporte, j'entends ce gouvernement qui est sa tâche propre, il fait en sorte qu'il so

25. Par le miracle notamment.

rappelle fort opportunément la remarque d'Alan Boase : « La pointe proprement dite pourrait se définir dans le sens le plus strict en di 27. F. ZUCCARO :op. cit.,I,7.

28. F. ZUCCARO :ibidem.

29. La distinction est celle de E. TESAURO :Il CannochialeAristotelico...,Torino. 1655,pp. 118-119.

30. -E. TESAURO :ibidem,p. 119.

31. P. DE L'ANCRE :Tableau de l'Inconstance et Instabilité de toutes choses,sec. éd.à

Paris chez la vesve Abel L'Angelier,M. DC.X, livre II, Discours IV, pp. 127 b et sq. Une des caractéristiques de cette espèce de folie est d'être liéeàl'inconstance.

32. -C'est la thèse queTESAURodéfend dans sonCannochiale.

Il est intéressant de la mettre en rapport avec l'emblématique que Tesauro pratiqua d'ailleurs et qu'il range parmi les « argutezze ».

33. Il est tiré duBois Sacré de Bomarzo,près de Viterbe, au nord de Rome, et n'estqu'un des nombreux éléments inédits que notre étudeamisàjour.

34. Souvenons-nous, par exemple, du jeu demot ficiniensurKa;).[ etKa;).6<;;àpropos de la Beauté.

35. Le danger defolie,Cf.supra, note 31.

36. -E. TESAURO :ibidem,parexemple cap. I,pp. 21et sq.

\section{AUTEUR}

\section{JEAN-MARIE WAGNER}

Historien de la philosophie 